\title{
Study of Serum Uric Acid Level in Patients with Systemic- Essential Hypertension in the Context of New 2017 ACC/AHA High Blood Pressure Clinical Practice Guideline
}

\author{
Atanu Ghosh¹, Debasish Pal², Sagar Samrat Debbarma³ ${ }^{2}$ Pranabesh Chakraborty ${ }^{4}$ \\ ${ }^{1}$ Department of Medicine, Agartala Government Medical College, G. B. Pant Hospital, Kunjaban, \\ Agartala, Tripura, India. ${ }^{2}$ Department of Medicine, Agartala Government Medical College, G. B. Pant \\ Hospital, Kunjaban, Agartala, Tripura, India. ${ }^{3}$ Department of Medicine, Agartala Government Medical \\ College, G. B. Pant Hospital, Kunjaban, Agartala, Tripura, India. ${ }^{4}$ Department of Medicine, Agartala \\ Government Medical College, G. B. Pant Hospital, Kunjaban, Agartala, Tripura, India.
}

\section{ABSTRACT}

\section{BACKGROUND}

Hypertension poses a challenge to public health as well as to the medical science. Final metabolite of purine in humans is uric acid, increased serum level of which is associated with cardio-renal risk, although serum uric acid (SUA) level appears to have different effects on blood pressure (BP), depending on level and how long subjects were exposed. We wanted to study the association between hyperuricemia and hypertension, in various aspects, in the context of new 2017 ACC/ AHA High Blood Pressure Clinical Practice Guidelines.

\section{METHODS}

The study was conducted in a tertiary care hospital, Agartala, Tripura. It is a case control study. SUA estimation was in 160 hypertensive patients and 160 normotensive controls, by using uricase method. SPSS version 24 was used for data entry and analysis. Chi square test and unpaired t test were used, wherever applicable.

\section{RESULTS}

Out of 320 subjects, who were included in the study, 160 subjects were hypertensive and 160 subjects were normotensive. There was strong association between hypertension and hyperuricemia. Hyperuricemia is more common in males and in patients with stage II hypertension.

\section{CONCLUSIONS}

The association between hyperuricemia and hypertension, has long been recognized. It remains unresolved whether the association of high uric acid level with high blood pressure is solely because of the underlying renal and metabolic abnormalities. Association of hyperuricemia and hypertension indicated that, hyperuricemia might be a target for the proposed therapeutic evaluation for the prevention of hypertension and provided impetus for the future design research work including interventional studies.

\section{KEY WORDS}

Serum Uric Acid, Hyperuricemia, Hypertension, 2017 ACC/ AHA High Blood Pressure Clinical Practice Guideline, Cardiovascular disease, Hypertensive, Normotensive
Corresponding Author:

Dr. Atanu Ghosh,

Dimsagar, Agartala-799001,

Tripura, India.

E-mail:dr.atanughosh01@gmail.com

DOI: $10.14260 /$ jemds/2020/82

Financial or Other Competing Interests: None.

How to Cite This Article:

Ghosh A, Pal D, Debbarma SS, et al. Study of serum uric acid level in patients with systemic-essential hypertension in the context of new 2017 ACC/AHA high blood pressure clinical practice guideline. J. Evolution Med. Dent. Sci. 2020;9(06):363367, DOI: 10.14260/jemds/2020/82

Submission 27-09-2019,

Peer Review 18-01-2020

Acceptance 24-01-2020,

Published 10-02-2020.

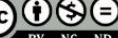




\section{BACKGROUND}

High blood pressure has a major impact on public health, and it is a problem to community and as well as for the physician. It is also challenging task for the medical science. Uric acid (UA) is a ring structure of cyclic compound of carbon, nitrogen, oxygen and hydrogen. Final metabolite of purines in humans is uric acid and increasing serum UA level is known to be associated with incident hypertension. ${ }^{1,2}$ The overall risk of incident hypertension (HTN) increased by $13 \%$ per $1 \mathrm{mg} / \mathrm{dl}$ increase in serum uric acid level and the risk was more evident in younger subjects, and in women. Although a serum uric acid of $6.8 \mathrm{ml} / \mathrm{dl}$ was used to define hyperuricemia in systemic review and meta-analysis prospective studies, all of the original studies that employed categorical serum urate levels have reported the risk of incident HTN increases with increasing levels of SUA. This data suggest that the relationship between serum uric acid (SUA) levels and HTN is likely to be linear, rather than being dependent on a specific cut point or threshold. The association was stronger among subjects $\gtrsim 40$ years old. No significant interaction between serum uric acid (SUA) and BMI (Body Mass Index) was observed. In a Japanese study, by Yuki Yokoi et al done on 26,442 males opined that serum UA is a predictor of hypertension (HTN) in young and middle Japanese males. The mechanism regarding inducing HTN by elevated SUA levels is an enigma and it remains elusive. Proposed implicated mechanisms for developing HTN by hyperuricemia are oxidative stress, inflammation, nitric oxide production impairment. Other reported mechanisms, for hyperuricemia related development of HTN are vascular endothelial dysfunction, vascular smooth muscle proliferation, and renin angiotensin system enhancement. ${ }^{2}$ Serum uric acid (SUA) is associated with high blood pressure and cardio-renal disease, ${ }^{3}$ although SUA appears to impart variable effects on blood pressure (BP) depending on level and how long the subject was exposed. ${ }^{4}$

In recent years, there has been an increase in prevalence of hyperuricemia, and the latter has attracted attention as an adult lifestyle-associated disease, with associated comorbidities like hypertension, diabetes and dyslipidaemia. ${ }^{5}$ Studies targeted young obese prehypertensive patients revealed the blood pressure reduction when they were treated with Allopurinol, an inhibitor of uric acid production and similar results were demonstrated when treated with Probenecid, an excretory accelerator of uric acid. These aforementioned opinions were derived from randomized placebo-controlled, double-blind, interventional study. Use of Allopurinol causing decrease in serum uric acid level is also associated with lowering high blood pressure, which was demonstrated by earlier meta-analysis. In one cross-sectional study conducted by Masanari Kuwabara, which targeted more than 90,000 subjects who attended St. Luke's International Hospital for a medical check-up elucidated that even when patients taking high blood pressure reducing medications and drugs decreasing high uric acid level, were excluded and the analysis was adjusted in regard to age, BMI, dyslipidaemia, diabetes, smoking and estimated glomerular filtration rate, there were 1.2 fold hike in prevalence of hypertension when serum uric acid levels were increased. By $1 \mathrm{mg} / \mathrm{dl}$. The quartile examination of serum uric acid levels showed that the group with the highest serum uric acid levels had 1.7 and 3.4 fold prevalence of HTN in men and women patients respectively, compared to the group with the lowest serum uric acid levels. 5 Westernized lifestyle and environment are most likely to be the cause for the increase in frequency in hyperuricemia and gout. ${ }^{6}$

Hypertension is one of the most challenging issues for public health, community as a whole and for the physicians. Its complication associated with mortality to the tune of 9.4 million patients, among 17 million deaths from cardiovascular disease yearly across the globe.7,8 Association of hyperuricemia with hypertension has been proposed from the findings and opinion of various studies. Serum uric acid levels were demonstrated to be an independent predictor of developing HTN. Early intervention of controlling serum uric acid was proposed to have effect to have delaying the progression of early HTN. Hyperuricemia increased the relative risk of hypertension by approximately $30 \%$ in men under 60 and by 2.6 -fold in women under 40.8 Though blood pressure (BP) controlling medicine to control the B.P., to the recommended level as per guidelines, number of uncontrolled hypertensive patients remained very high. To prevent complications of high B.P., such as coronary heart disease, stroke and renal disease, risk factors of uncontrolled B.P. should be considered when establishing proper, guidelines recommended B.P. control strategies. Research on uncontrolled HTN needs to be conducted to identify the relationship between hyperuricemia and cardiovascular events. ${ }^{8}$ To define hyperuricemia, the serum urate level should be $\geq 6.8 \mathrm{mg} / \mathrm{dl}$, that is the limit of the urate solubility at physiological temperature and $\mathrm{pH}^{9}$ Comprehensive evaluation for symptoms and signs of cardiovascular disease as well as aggressive management of the cardiovascular risk factors like, reduction of weight and alcohol intake, control of blood pressure, glucose and lipids should be undertaken in patients with hyperuricemia or gout. 10

Though there is ambiguity for long in regard to the role of serum uric acid (SUA) as a cause for hypertension (HTN) and cardiovascular disease (CVD), there is a growing interest, implicating SUA as an independent risk factor for incident HTN, based on numerous prospective studies conducted in the United State, China, Italy and Japan. ${ }^{11,12,13,14,15,16,17}$ Association of increased blood pressure with SUA was noted first by Frederick Mohamed in 1870s. ${ }^{18,19}$ In different ethnic groups, a continuous relationship between SUA and B.P. was observed in African-Americans and whites, in Asians including Koreans. ${ }^{20}$ In adults CVD is the most common form of target organ related damage as far as HTN is concerned. The prevalence of HTN grows higher with aging, resulting in an increase in morbidity and mortality through various events such as myocardial infarction, heart failure, stroke and renal failure. $20,21,22,23,24$ Hyperuricemia from various study related evidence has been proposed to have a linkage with HTN. Independent predictor for developing HTN were demonstrated to have related to SUA levels. ${ }^{25,26,27}$

In addition to HTN, hyperuricemia substantially associated with cardiovascular disease, stroke and decrease in kidney function. ${ }^{28,29}$ Present study aims to assess the relation between hypertension as per new 2017 ACC/AHA new guideline and hyperuricemia in a tertiary care referral hospital in Agartala, Tripura, ${ }^{30}$ in both sex related comparability, and variability. 


\section{METHODS}

This is a case control study. As per Whelton PK, et al. New 2017 ACC/AHA High Blood Pressure Clinical Practice Guideline Classification, 30160 hypertensive patients were included in the study and 160 normotensive patients were included as control. Sample size was taken based on the convenience of the study. Serum Uric Acid (SUA) estimation was done by uricase method among 160 cases of hypertensive patients and 160 controls of normotensive patients, in our tertiary care health institution in Agartala, Tripura from the month of November 2017 to October 2018. Patients who were on drugs/ medications, antihypertensive agents, which had the propensity to alter the serum acid level, were not included in this study. Patients with renal failure, secondary hypertension, pregnancy, heart failure, suffering from conditions/ diseases which could likely to affect the SUA level, unwilling to participate in the study were also excluded from this study.

Ethical approval was taken from Institutional Ethical Committee.

\section{Statistical Analysis}

Data entry and analysis was done in SPSS version 24. Categorical data was expressed in means. Chi square test and unpaired t test were used wherever applicable to find out any association between variables. Odds ratio was calculated along with $95 \%$ confidence interval. p-value $<0.5$ was considered as significant.

\section{RESULTS}

320 subjects were enrolled in this case control study, where 160 subjects (hypertensive) as cases and 160 subjects (normotensive) as control. In hypertensive group 66.3\% were male and 33.8 percent were female. In hyperuricaemic patients $39.4 \%$ had stage I hypertension and $60.0 \%$ patients belonged to stage II hypertension. Among control normotensive group (160 patients, 103 male and 57 females) SUA varied from 2.10 to 9.90 (mean 4.9377, SD 1.538).

\begin{tabular}{|c|c|c|c|c|}
\hline \multirow{2}{*}{ Hypertension } & \multicolumn{2}{|c|}{ Hyperuricaemia } & \multirow{2}{*}{$\begin{array}{c}\text { Significant } \\
\text { (p value) }\end{array}$} & \multirow{2}{*}{$\begin{array}{c}\text { Odds Ratio } \\
(95 \% \mathrm{CI})\end{array}$} \\
\hline & Yes & No & & \\
\hline Yes & $76(47.5 \%)$ & $84(52.5 \%)$ & Chi Square $=50.67$ & \multirow{3}{*}{7.13} \\
\hline No & $18(11.2 \%)$ & $142(88.8 \%)$ & df $1 . p$ value $=0.00$ & \\
\hline $\begin{array}{l}\text { Hyperuricemia } \\
(\mathrm{p}=0.00)\end{array}$ & significantly & ssociated wit & & \\
\hline
\end{tabular}

\begin{tabular}{|c|c|c|c|c|}
\hline & Minimum & Maximum & Mean & Std. Deviation \\
\hline SBP & 130 & 180 & 149.35 & 12.943 \\
\hline DBP & 80 & 110 & 90.77 & 7.118 \\
\hline URIC Acid & 2.50 & 12.40 & 6.5516 & 1.61575 \\
\hline Age & 19 & 79 & 53.73 & 11.304 \\
\hline $\begin{array}{l}\text { Table 2 Mean age of cases was 53.73 } \pm 11.30, \text { mean SBP was } 149.35 \pm 12.94, \text { and } \\
\text { mean DBP was 90.77 } \pm 7.118 \text { mean uric acid 6.551 } \pm 1.615 .\end{array}$ \\
\hline $\begin{array}{l}\text { SBP=Systolic Blood pressure. DBP=Diastolic Blood pressure. (in mm of Hg) } \\
\text { N=Number of patients/controls. Std. Deviation=Standard Deviation. }\end{array}$ \\
\hline \multicolumn{5}{|c|}{ Table 2. Descriptive Data of Cases (n=160) } \\
\hline
\end{tabular}

\begin{tabular}{|c|c|c|}
\hline & Serum Uric Acid & Significance \\
\hline Hypertensive & $6.55 \pm 1.615$ & $\mathrm{~T}=9.22$ \\
$\mathrm{P}=0.00$ \\
\hline Non-hypertensive & $4.93 \pm 1.538$ & mean serum uric acid \\
\cline { 1 - 2 } Table 3 showing that there is significant difference between & mevels among hypertensive and non-hypertensive subjects $( \pm 9.22, \mathrm{p}<0.05)$ \\
\hline \multicolumn{2}{|c|}{ Table 3. Distribution of Serum Uric Acid among the Two Groups } \\
\hline
\end{tabular}

47.5\% hypertensive patients had hyperuricemia whereas $11.2 \%$ normotensive patients had high serum uric acid level. Hyperuricemia patients were more in number in stage II hypertensive as compared to stage I hypertensives. Male predominance was observed among hypertensive subjects with high uric level. There was strong association between hypertension and high serum uric acid level. Out of 160 hypertensive (case) and 160 non-hypertensive patients (control), unpaired t test was used for statistical analysis, where $\mathrm{t}=8.941(\mathrm{p}=0.000), 1.23 \pm 1.93$ at $95 \% \mathrm{Cl}$. These are statistically significant to show strong association of hyperuricemia with hypertension.

\section{DISCUSSION}

Hyperuricaemia is a change in metabolic milieu related problem, and it has become increasingly common across the globe and its linkage as a cause with hypertension has been observed for over 130 years ${ }^{31}$. Our study findings also consistent with these findings. In adults with hyperuricemia and hypertension, there is a dearth of investigative information to characterize the treatment related advice for the reduction of serum urate in the management of hypertension. ${ }^{29}$

The association of hyperuricemia with hypertension (HTN) has long been recognized. ${ }^{32,33}$ It remained unanswered whether the association of hyperuricemia with HTN is solely because of the underlying renal abnormality and change in metabolic milieu. Decreased renal blood flow and decreased tubular secretion of uric acid have been associated with 34,35 high serum uric acid with hypertension. Study result came out regarding high incidence of hyperuricemia in hypertensives also revealed in other studies done by Sujeet Raina, Surender Thakur, Surinder, Thakur et $\mathrm{al}, 36$ the findings which were similar with our study. Hyperuricemia is really not uncommon, the prevalence of which were reported between $2.6 \%$ and $47.2 \%$ in various populations. As far as study related to the select nomad population of Rajasthan, India, the prevalence of high SUA was found to be $13.5 \%$. Hyperuricemia was more frequent found in male $(14.4 \%)$ as compared to female (12.8\%).36 In our study we could find as compared to normotensives, hyperuricemia is more prevalent in hypertensive subjects. Patients with increased uric acid level may be more responsive to the uric acid lowering treatment, early in the course of their disease. ${ }^{37}$ The cumulative incidence rate of hypertension was significantly higher in subjects with hyperuricemia than those of without hypertension, in normotensive $(5.6 \%$ versus $2.6 \%)$ and prehypertensive subjects (30.7\% versus $24.0 \%$ ). Hyperuricemia increased the risk of hypertension by $=35 \% .^{38}$ In our study we could find as compared to normotensives, hyperuricemia is more prevalent in hypertensive subjects.

For determining the causal role of SUA in development of hypertension, Mazzali $\mathrm{M}$, et al demonstrated a hike in SUA followed by B.P elevation. Via a crystal-independent mechanism in rat models. ${ }^{42}$ In a research article Ji Wang, et al mentioned that multiple observational studies were contemplated to reveal the association between uric acid and HTN, but ambiguity remains, regarding this matter. Elevated 
uric acid levels are often linked with established traditional cardiovascular risk factors but it remained unresolved whether uric acid is the cause or consequence of HTN. Studies relating uric acid as an independent risk factor did not sufficiently control for other known risk factors. Understanding regarding the causal role uric acid in causation of HTN is not fully addressed. Several events has led to the reappraisal of the role of uric acid in HTN. Studies using animal models and red cell cultures have identified mechanisms by which uric acid might induce HTN via reducing nitric oxide, activation of renin-angiotensin system, causing smooth muscle proliferation and production of various inflammatory mediators. Some prospective cohort studies that have controlled for multiple risk factors suggest that uric acid may be an independent risk factor for high blood pressure development and preliminary clinical trials reported benefits from reducing uric acid in hypertensive patients, but some reported inconsistent conclusions ${ }^{39}$. In our study we could find a strong association between hyperuricemia and HTN. Investigation of the population of the Hypertension-Nephritis Clinic of the Presbyterian Hospital in New York City confirms the observation among patients with either primary or renal HTN, treated and untreated, that there is an increased incidence of hyperuricaemia. ${ }^{40}$

Feigh DI., in an article related to hyperuricemia and hypertension, commented regarding consideration of uric acid as a potential endangering factor for hypertension and cardiovascular disease, over the past century. However, only in the past decade, mechanistic link was supported from the study-inferences of animal models and clinical trials. As revealed from animal model studies, 2-phase mechanism suggested, for the development of HTN in hyperuricemia. The suggestions were that, implication of uric acid in induction of acute vasoconstriction by activation hormone system like renin- angiotensin-aldosterone system, followed by vascular smooth muscle cells uptake of uric acid leading to cellular proliferation and secondary arteriosclerosis that impairs pressure sodium excretion. The acute HTN remains uric acid dependent and sodium independent whereas chronic HTN becomes uric acid independent and sodium dependent. It was demonstrated in small clinical trials involving adolescents, that reduction of uric acid can decrease blood pressure in newly diagnosed hypertensive patients. Although more researches are definitely indicated, from the available data it may well be opined that in relation to some cases of early onset HTN, uric acid is likely to be the cause. ${ }^{37}$

In 1897 it came to notice that patients from gouty families had preponderance of HTN and the uric acid was implicated in the development of hypertension. Most of the subsequent cross-sectional and longitudinal studies demonstrated that serum uric acid as a predictor of HTN. In this issue of high blood pressure and high uric acid level, Kuwabara et al extended our knowledge by showing that hyperuricaemia played a significant role in the progression of prehypertension to hypertension and provided important message that approximately a third pre-hypertensive and hyperuricemia subjects would develop HTN in five years and suggested a potential target population for future trials.5,38 In a clinical study by Feigh DI, et al. commented that reduction of high serum uric acid by medications was beneficial for lowering blood pressure in adolescents with newly diagnosed HTN ${ }^{41}$. In our study we could find high uric acid level patients are more in Stage - II hypertensive patients as compared to Stage -I hypertensive patients and hyperuricemia is more prevalent in male hypertensive patients as compared female patients. Larger multiple trials with more number of patients are needed to support our findings.

\section{CONCLUSIONS}

In our study we could find increased association of hyperuricemia with hypertensive patients as compared to normotensive subjects. Among hypertensive patients high uric acid levels were observed in stage II hypertensives as compared to stage I patients. Male hyperuricaemic preponderance is seen in hypertensive patients. On the basis of our study findings, it may well be opined that SUA level may be used as a biochemical marker to determine the duration and severity of high blood pressure. We need larger trials to comment on the early appearance of hyperuricemia to be a definitive indicator of later development of hypertension, and in adults with essential hypertension the comorbidity of hyperuricemia is not really uncommon, ${ }^{29}$ although high uric acid level predicted incidence of hypertension and prehypertension in most cohort studies, the causal relationship between hypertension and high uric acid level had not been fully established. Association of high uric acid level and hypertension indicated that, hyperuricemia might be a target for proposed therapy for the prevention of hypertension.

\section{REFERENCES}

[1] Grayson PC, Kim SY, LaValley M, et al. Hyperuricemia and incident hypertension: a systemic review and metaanalysis. Arthritis Care Res (Hoboken) 2011;63(1):10210.

[2] Yokoi Y, Kondo T, Okumura N. Serum uric acid as a predictor of future hypertension: stratified analysis based on body mass index and age. Prev Med 2016;90:201-6.

[3] Zoccali C, Mallamaci F. Uric acid, hypertension and cardiovascular and renal complications. Curr Hypertens Rep 2013;15(6):531-7.

[4] Feig DI., Madero M, Jalal DI, et al. Uric acid and the origins of hypertension. J Pediatr 2013;162(5):896-902.

[5] Kuwabara M. Hyperuricemia, cardiovascular disease and hypertension. Pulse (Basel) 2016;3(3-4):241-52.

[6] Guidelines Revision 3 Committee, JSNM: Guidelines for the management of hyperuricemia and gout. $2^{\text {nd }}$ edn. Tokyo Medical Review 2010.

[7] Lim SS, Vos T, Flaxman AD, et al. A comparative risk assessment of burden of disease and injury attributable to 67 risk factors and risk factors clusters in 21 regions, 1990-2010: a systemic analysis for the Global Burden of Disease Study 2010. Lancet 2012;380(9859):2224-60.

[8] Cho J, Kim C, Kang DR, et al. Hyperuricemia and uncontrolled hypertension in treated hypertensive 
patients: K-MetS Study. Medicine (Baltimore) 2016;95(28):e4177.

[9] Neogi T. Clinical practice. Gout. $N$ Eng J Med 2011;364(5):443-52.

[10] Fravel MA, Ernest ME. Gout and hyperuricemia. Chapter 93. In: DiPiro JT, Talbert RL, Yee GC, et al. eds. pharmacotherapy: a pathophysiologic approach. $10^{\text {th }} \mathrm{edn}$. New York: McGraw-Hill Medical 2017.

(https://accesspharmacy.mhmedical.com/content.aspx? sectionid=14606925\&bookid=1861)

[11] Yang T, Chu CH, Bai CH, et al. Uric acid concentration as a risk marker for blood pressure progression and incident hypertension: a Chinese cohort study. Metabolism 2012;61(12):1747-55.

[12] Loeffler LF, Navas-Acien A, Brady TM, et al. Uric acid level and elevated blood pressure US adolescents: National Health and Nutrition Examination Survey, 1999-2006. Hypertension 2012;59(4):811-7.

[13] Sundstrom J, Sullivan L, D'Agostino RB, et al. Relations of serum uric acid to longitudinal blood pressure tracking and hypertension incidence. Hypertension 2005;45(1):28-33.

[14] Nagahama K, Inoue T, Iseki K, et al. Hyperuricemia as a predictor of hypertension in a screened cohort in Okinawa, Japan. Hypertens Res 2004;27(11):835-41.

[15] Gaffo AL, Jacobs DR Jr, Sijtsma F, et al. Serum urate association with hypertension in young adults: analysis from the Coronary Artery Risk Development in Young Adult Cohort. Ann Rheum Dis 2013;72(8):1321-7.

[16] Zhang W, Sun K, Yang Y, et al. Plasma uric acid and hypertension in a Chinese community: prospective study and meta-analysis. Clin Chem 2009;55(11):2026-34.

[17] Cicero AFG, Salvi P, D'Addato S, et al. Association between serum uric acid, hypertension, vascular stiffness and subclinical atherosclerosis: data from the Brisighella Heart Study. J Hypertens 2014;32(1):57-64.

[18] Swales JD. Manual of hypertension. Oxford: Blackwell Science 1995.

[19] Neki NS, Tamilmani. A study of serum uric acid level in essential hypertension. JIMSA 2015;28(1):13.

[20] Lee JJ, Ahn J, Hwang J, et al. Relationship between uric acid and blood pressure in different age groups. Clinical Hypertension 2015;21:14.

[21] Feigh DI, Johnson RJ. Hyperuricemia in childhood primary hypertension. Hypertension 2003;42(3):247-52.

[22] Coresh J, Wei GL, McQuillan G, et al. Prevalence of high blood pressure and elevated serum creatinine level in the United States: findings from the third National Health and Nutrition Examination Survey (1988-1994). Arch Intern Med 2001;161(9):1207-16.

[23] Park JK. Epidemiology of hypertension. J Korean Soc Hypertens 1995;1:6-17.

[24] Kim JS, Lee HC, Yoo WS. Mean blood pressure, prevalence and epidemiologic characteristics of hypertension among representative Korean adult population. J Korean Soc Hypertens 1998;4:89-98.

[25] Jossa F, Farinaro E, Panico S, et al. Serum uric acid and hypertension: the Olivetti heart study. J Hum Hypertens 1994;8(9):677-81.
[26] Brand FN, McGee DL, Kannel WB, et al. Hyperuricemia as a risk factor of coronary heart disease: the Framingham study. Am J Epidemiol 1985;121(1):11-8.

[27] Kansui Y, Ohtsubo T, Goto K, et al. Association of serum uric acid with blood pressure in Japanese men. Crosssectional study in work-site group. Circ J 2011;75(12):2827-32.

[28] Tangri N, Weiner DE. Uric acid, CKD and cardiovascular disease: confounders, culprits and circles. Am J Kidney Dis 2010;56(2):247-50.

[29] Whelton A. Hyperuricemia \& hypertension. a confluence of concepts. Hypertension 2012;60(5):1112-3.

[30] Whelton PK, Carey RM, Aronow WS, et al. 2017 ACC/AHA/AAPA/ABC/ACPM/AGS/APhA/ASH/ASPC/NA /PCNA Guideline for the Prevention, Detection, Evaluation, and Management of High Blood Pressure in Adults: Executive Summary: A Report of the American College of Cardiology/American Heart Association Task Force on Clinical Practice Guidelines. Hypertension 2018;71(6):1269-324.

[31] Mahomed FA. On chronic Bright's disease and its essential symptoms. Lancet 1879;113(2899):399-401.

[32] Dollery CT, Duncan H, Schumer B. Hyperuricemia related to treatment of hypertension. BMJ 1960;2(5202):832-5.

[33] Perlstein TS, Gumieniak O, Williams GH, et al. Uric acid and the development of hypertension: the normative aging study. Hypertension 2006;48(6):1031-6.

[34] Messereli FH, Frohlich ED, Dreslinski GR, et al. Serum uric acid in essential hypertension: an indicator of renal vascular involvement. Ann Intern Med 1980;93(6):81721.

[35] Tykarski A. Evaluation of renal handling of uric acid in essential hypertension: hyperuricemia related to decreased urate secretion. Nephrob 1991;59(3):364-8.

[36] Raina S, Thakur S. Serum uric acid levels in hypertensive patients with and without metabolic syndrome in hills of Himachal Pradesh, India. Journal of Obesity and Metabolic Research 2014;1(3):156-8.

[37] Feig DI. Hyperuricemia and hypertension. Adv Chronic Kidney Dis 2012;19(6):377-85.

[38] Cheng YB, Yan Li. Hyperuricemia does it matter for the progression from prehypertension to hypertension? Hypertension 2018;71(1):66-7.

[39] Wang J, Qin T, Chen J, et al. Hyperuricemia and risk of incident hypertension: a systemic review and metaanalysis of observational studies. PLoS One 2014;9(12):e114259.

[40] Cannon PJ, Stason WB, Dermartini FE, et al. Hyperuricemia in primary and renal hypertension. N Eng J Med 1966;275(9):457-64.

[41] Feigh DI, Soletsky B, Johnson RJ. Effect of allopurinol on blood pressure of adolescents with newly diagnosed essential hypertension: a randomized trial. JAMA 2008;300(8):924-32.

[42] Mazzali M, Hughes J, Kim YG, et al. Elevated uric acid increases blood pressure in the rat by a novel crystalindependent mechanism. Hypertension 2001;38(5):1101-6. 